\title{
Interkulturalität und multilinguale literarische Werke als Basis für ein Konzept der Mehrsprachigkeit im DaF-Unterricht
}

\section{Pavel Váňa}

This article would like to present a concept for a multilingual literature teaching. The first part examines the general possibilities related to this approach, whereas the second part shows practical methods in reference to an international Czech and German literary workshop.

German literature - multilingual literature teaching - German literature - Intercultural communicative competence

Dieser Artikel befasst sich mit dem Konzept eines mehrsprachlichen Literaturunterrichts. Im ersten Teil werden die Möglichkeiten eines Literaturunterrichts mit mehrsprachlichem Akzent angeführt. Der zweite Teil ist einigen praktischen Beispielen der Berücksichtigung von Mehrsprachigkeit im Literaturunterricht gewidmet, wobei ein deutsch-tschechischer Literaturworkshop vorgestellt wird.

Deutsche Literatur - Literaturunterricht - Texterschließung - Mehrsprachigkeit epochenzentriert - deutsch-tschechischer Literaturworkshop

\section{Einleitung}

Der folgende Beitrag ist dem Thema „Literatur im DaF-Unterricht“ gewidmet, der sich bei der Behandlung von literarischen Werken oder Epochen auf fremdsprachliche (L-2) Vorkenntnisse der Lerner stützt.

Als erstes wird das Phänomen der Mehrsprachigkeit im Kontext des Literaturunterrichts erfasst und dessen Verwendung in zwei kurz skizzierten Modellen einer Unterrichtsstunde besprochen. Obwohl in Tschechien die Mehrsprachigkeit (gesetzt den Fall, dass es sich um mehr als zwei Sprachen handelt) vordergründig als die Kombination von L-1: Muttersprache, L-2: Englisch, L-3: Deutsch (statt Deutsch eventuell eine andere Fremdsprache) betrachtet wird, ist mit Mehrsprachigkeit bei den meisten Schlussfolgerungen in diesem Artikel generell eine offenere Sprachenfolge gemeint, und zwar die allgemeine Konstruktion Muttersprache-Fremdsprache-1 - Fremdsprache-2.

\section{Die Mehrsprachigkeit}

Das Phänomen der Mehrsprachigkeit wird seit mehreren Jahrzehnten wissenschaftlich untersucht (vgl. z. B. Griesmayer 2000; Winterstein 2000; Belke 2008 u. a.), wobei 
viele Autoren ${ }^{1}$ bei ihren Ausführungen auf die von Els Oksaar formulierte Definition der Mehrsprachigkeit zurück greifen:

„Mehrsprachigkeit“ definiere ich als funktional. Sie setzt voraus, dass der Mehrsprachige in den meisten Situationen ohne weiteres von der einen Sprache zur anderen umschalten kann, wenn es nötig ist. Das Verhältnis der Sprachen kann dabei durchaus verschieden sein - in der einen kann, je nach der Struktur des kommunikativen Aktes, u. a. Situationen und Themen, ein wenig eloquenter Kode, in der anderen ein mehr eloquenter Kode verwendet werden." (Oksaar 1980: 43)

Von dieser allgemein akzeptierten Definition ausgehend, wird im folgenden Beitrag vor allem der Akzent auf die Unterschiedlichkeit im Verhältnis der zwei Sprachen gesetzt, die im kommunikativen Akt verwendet werden, wobei das Umschalten von der einen auf die andere Sprache, das „ohne weiteres“ geschehen soll, bei den DaFLernern erst in späteren Phasen ihres Studiums der L-2 (L-3) vorauszusetzen ist.

Um das Verhältnis zwischen Mehrsprachigkeit und Literatur zu verdeutlichen, werden die vielen Unterrichtsmodelle im Bereich des Faches fremdsprachliche Literatur auf zwei elementare Formen reduziert: den chronologisch gestalteten und den textzentrierten Literaturunterricht.

\section{Der chronologisch aufgebaute Literaturunterricht}

Der Literaturunterricht kann auf zwei unterschiedliche grundlegende Konzepte zurückgeführt werden (vgl. Abraham und Kepser 2009: 129-184), von denen das erste, chronologisch aufgebaute davon ausgeht, dass die zu erwerbenden Kenntnisse im Rahmen eines epochenzentrierten Modells erzielt werden können. Der Schwerpunkt liegt dabei auf den typischen Merkmalen aller wichtigen Teilbereiche der Literaturgeschichte. Die literarischen Epochen bilden jeweils ein geschlossenes Modul, in dem alle einzelnen relevanten Komponenten von Belang sind: der Epochenbegriff, der historische und kulturelle Hintergrund, sowie die politische Entwicklung und die damit verbundenen (oft schwerwiegenden) sozialen Strukturveränderungen, zu denen es während der behandelten literarischen Epoche gekommen ist. Zu diesem breit aufgefassten Umfeld werden dann auch die relevanten Autoren und ihre literarischen Werke gezählt, die unter Berücksichtigung dieses komplexen Rahmens besprochen werden.

Bei dem chronologisch aufgebauten Literaturunterricht bilden die einzelnen literarischen Epochen einen kontinuierlichen Hintergrund, vor dem die wichtigsten Merkmale, poetologischen Konzepte und Autoren vorgestellt und ihre Werke

1 Dies betrifft z. B. folgende Autoren, die sich mit dem Thema der Mehrsprachigkeit auseinandersetzen und von der Definition von Oksaar bei ihren Schlussfolgerungen ausgehen: Claudia Maria Riehl (2009), Nicola Rück (2009), Ursula Neumann (2009) u. a. 
analysiert werden. Das gesamte Bild der Epoche entsteht jeweils nach denselben oder ähnlichen zeit- oder kulturgeschichtlichen Impulsen. Und dennoch heben sich die in unterschiedlichen Sprach- und Kulturräumen entstandenen Epochenbilder voneinander ab, wobei typische Unterschiede zwischen den Kulturräumen oft an eben diesen Unterschieden der Epochenbilder festgemacht werden. Jede literarische Epoche spiegelt nicht nur die kulturellen, gesellschaftlichen oder politischen Besonderheiten des jeweiligen Landes oder Sprachgebiets wider, sondern weist im Vergleich mit der Realität der fremdsprachlichen Gebiete unzählige Überschneidungen, parallele Entwicklungen, Beeinflussungen, Entlehnungen und Ähnlichkeiten auf.

\section{Der textbezogene Literaturunterricht}

Bei diesem zweiten Unterrichtstyp steht die Arbeit mit dem literarischen Text im Vordergrund und die mit den Epochencharakteristika zusammenhängenden Informationen bilden einen ergänzenden Rahmen, der meist als eine sekundäre Quelle von zusätzlichen Informationen benutzt wird. Auf die Daten aus diesem Bereich wird nur dann verwiesen, wenn sie sich auf eine Textstelle oder eine in dem besprochenen literarischen Werk untersuchte Problematik beziehen. Die textnahe Arbeit mit den Werken der deutschsprachigen Literatur, gestützt auf die L-2 Kenntnisse der Studierenden, stellt eine andere relevante Vorgangsweise dar, wie man interkulturellen Ansätzen im Literaturunterricht gerecht werden kann.

Zurückgreifend auf die L-2-Kenntnisse kann man die Stoffgeschichte des „Faust“ verfolgen - von der ersten datierten Buchdruckfassung aus dem Jahre 1587 „Historia von D. Johann Faustus“ zur Bearbeitung desselben Stoffes durch Christopher Marlowe aus dem Jahre 1589 unter dem Titel „Die tragische Historie vom Doktor Faustus“. Diese wurde von den englischen Gruppen des Wandertheaters aufgegriffen, bearbeitet und nach Deutschland gebracht. Faust wurde zur komischdämonischen Hauptfigur der verschiedensten Darstellungsformen, wie z. B. Ballett, Puppentheater oder Wandertheater. In den 20er Jahren des 18. Jahrhunderts waren Faustpantomimen von John Rich und John Thurmond beliebt. Die Bearbeitung des Stoffes durch J. W. von Goethe wird dann in der Unterrichtsstunde als der Höhepunkt der literarischen Auseinandersetzung mit diesem Stoff präsentiert.

Ähnliche Vorgehensmuster könnte man u. a. am Beispiel des Hamlet-Stoffes entwickeln. In dieses Umfeld gehört auch die Arbeit mit einzelnen literarischen Werken, in denen die Mehrsprachigkeitskomponente stark vertreten ist und für die ein spezieller literarischer Kanon erstellt werden muss. 


\section{Der Literaturunterricht im Fach Deutsch als Fremdsprache nach Englisch}

Um einen differenzierten Einblick in die einzelnen Epochen der deutschsprachigen Literaturgeschichte $\mathrm{zu}$ gewinnen, ist es empfehlenswert, sich mit einem der Standardwerke der einschlägigen Literaturgeschichte auseinanderzusetzen. ${ }^{1}$ Der Deutsch-Lernende wird in mehreren Etappen der Literaturgeschichte feststellen können, dass es bereits in der ältesten Zeit gemeinsame europäische Kulturräume und Kulturentwicklungen gab - gemeint sind sowohl die Heldenlieder, als auch die heidnischen schriftlichen Denkmäler oder die ersten literarischen Belege der Christianisierung des Abendlandes. Auf der chronologischen Achse treten diese kulturellen Berührungspunkte ziemlich deutlich ans Licht, etwa bei der Bearbeitung der Stoffe des höfischen Epos (des Parzival-Stoffes, des Tristan-Stoffes u. a.). In der späteren Zeit kann man in der Art einer Vernetzung zwischen der Ideenwelt der europäischen Reformation und den Werken und Taten deren Vertreter - von John Wiclef oder Johannes Hus und den bedeutendsten Vertretern der Schweizer Reformation, Johannes Calvin und Ulrich Zwingli, bis hin zur deutschen Reformation und nicht zuletzt zu Martin Luther - viele Gemeinsamkeiten, aber auch gravierende Unterschiede feststellen.

Man kann weiter die unzähligen Berührungspunkte der englischen und deutschen kulturellen Entwicklung der Jahre nach dem Dreißigjährigen Krieg verfolgen. Dies gilt insbesondere für die Epoche des „rationalen“ 18. Jahrhunderts, wo man im deutschen kulturellen Leben neben einem englischen zugleich auch einen französischen Einfluss feststellen kann, wobei diese Einflüsse nicht immer positiv gewertet wurden. ${ }^{2}$ Auch der Epochenbegriff der Klassik kann ohne seinen interkulturellen Hintergrund nicht vollständig definiert werden. Im Vergleich mit anderen europäischen Ländern wird man z. B. die zeitliche Differenz zwischen der französischen und deutschen Klassik nicht nur als gegebene Tatsache hinnehmen, sondern man wird ihre Ursachen feststellen wollen, um die Logik dieser Abweichung besser verstehen zu können.

Ohne die Erwähnung des „langen“ 19. Jahrhunderts (1800-1914) und des „kurzen“ 20. Jahrhunderts (1914-1989) wäre diese Aufzählung der Ähnlichkeiten von literarischen Entwicklungen in den verschiedenen Kulturräumen nicht vollständig. Im 19. Jahrhundert steht die deutsche literarische Szene unter einem sehr starken französischen und englischen Einfluss. In der deutschen Sprachrealität der ersten Hälfte des 19. Jahrhunderts gibt es genug klare Signale einer deutschenglischen Affinität. Um auf literarischem Boden zu bleiben, kann man die außerordentlich starke deutsche Rezeption der Werke der englischen Romantiker

1 Vgl. z. B. Magris (1966), Martini (1984), Žmegač (1978).

2 Vgl. z. B. die Kritik der Fremdtümelei bei den Vertretern der deutschen Sprachgesellschaften im 17. Jahrhundert (Wilpert 2001: 774-775). 
Percy Bysshe Shelley, John Keats, George Gordon Byron u. a. feststellen oder die anhaltende intensive Auseinandersetzung mit dem Schaffen Shakespeares zur Kenntnis nehmen, die in der Epoche der deutschen Romantik ihren vorläufigen Höhepunkt erreicht.

Im Verlauf des 19. Jahrhunderts tritt in England immer deutlicher die positive Resonanz der Werke Goethes und umgekehrt in Deutschland die starke Rezeption von literarischen Werken der englischen realistischen Schule - Charles Dickens, Thomas Hardy, William Thackeray u. a. - in den Vordergrund. Für die deutschfranzösische Verbundenheit gab es im 19. Jahrhundert im politischen Bereich (besonders nach der Julirevolution) kaum noch einen gemeinsamen Raum, um so stärker manifestierte sie sich in der Zeit der Romantik und des Vormärz auf dem Gebiet der Literatur. Es reicht, wenn man einschlägige Daten aus der Biographie von Madame de Staël, A. W. Schlegel, Adalbert von Chamisso, E. T. A. Hoffmann, H. Heine, A. von Platen u. a. anführt, um zu dokumentieren, wie eng die Kulturszene des deutschen und französischen Sprachgebiets im 19. Jahrhundert verbunden war.

\section{Ein Beispiel der praktischen Umsetzung von Mehrsprachigkeit im Literaturunterricht}

Dieser Teil des Beitrags ist der praktischen Möglichkeit der Einbeziehung von L-3-, bzw. L-2-Lernern in die kreativen Prozesse des Literaturunterrichts und ihrer Beteiligung an relevanten Verfahren der Texterschließung gewidmet, die unter Berücksichtigung von interkulturellen ${ }^{1}$ Ansätzen im Rahmen des Literaturunterrichts erfolgt.

Seit mehr als zehn Jahren werden am Lehrstuhl für deutsche Sprache und Literatur der Pädagogischen Fakultät der Masaryk-Universität internationale Literaturseminare veranstaltet, die zusammen mit dem Lehrstuhl Deutsch als Fremdsprachenphilologie der Universität Regensburg in der Regel zweimal jährlich stattfinden. Die Englisch-Kenntnisse der deutschen Teilnehmer und Teilnehmerinnen sind meistens als B1-B2 nach der Skala des GERR einzustufen und Ähnliches gilt auch für die tschechischen Studierenden, die an den Seminaren teilnehmen. Auf diese Tatsache soll bei der Gestaltung des Seminarplans in Zukunft größerer Wert gelegt werden: Bei der Zusammenstellung des Lesekanons für das Seminar wird die bisher ausschließlich tschechisch-deutsche oder mährisch-deutsche Perspektive um Schwerpunkte erweitert, die auf Einflüsse einer dritten Sprache zurückgeführt werden können.

Während der mehr als zehnjährigen Geschichte der internationalen Seminare variierten die Themen von einer breit aufgefassten Problematik bis hin zu Seminaren,

1 Im Bereich der Interkulturalität gibt es mehrere Fachbegriffe, die in folgender Quelle klassifiziert werden: Multikulturalität, Interkulturalität, Transkulturalität und Plurikulturalität. http://www.ikud. de/Multikulturalitaet-Interkulturalitaet-Transkulturalitaet-und-Plurikulturalitaet.html (2. 4. 2012). 
die sich mit relevanten Werken eines ausgewählten Autors befassen. Die folgende kurze Aufzählung der Seminarthemen der letzten zehn Jahre verrät eine gewisse Themenvielfalt, der interkulturelle und nicht zuletzt mehrsprachliche Hintergrund ist jedoch deutlich erkennbar:

- Das erste Seminar war dem Thema "Was ist Europa“ gewidmet. Die Autoren der Beiträge haben sich nicht nur mit der geographischen Komponente der Frage nach einem europäischen Kontext auseinandergesetzt, sondern auch damit, welche kulturellen, vor allem literarischen Phänomene als ein gemeinsames europäisches Erbe empfunden werden können. Für dieses Seminar wurden den Teilnehmern/innen zwei kanonische Werke empfohlen: Ach, Europa von Hans Magnus Enzensberger und Im blinden Winkel. Nachrichten aus Mitteleuropa von Christoph Ransmayr.

- Im folgenden Seminar Visionen von Europa wurden die deutsch-tschechischen Stereotypen und ihre Chronologie aus einer erweiterten Perspektive (15.-20. Jahrhundert) diskutiert.

- Das erste im engeren Sinne literarische Seminar hatte das Romanwerk Joseph Roths und Roths Festhalten an der Tradition des habsburgischen Vielvölkerstaates zum Thema: „Joseph Roth und der Untergang der Habsburger Monarchie“.

- Durch die Teilbereiche des Seminars "Poetik der Landschaft“ wurde der deutsch-mährische Bezugsrahmen am Beispiel der literarischen Werke von Ferdinand von Saar, Jacob Julius David und Marie von Ebner-Eschenbach veranschaulicht.

- Im Seminar „Die Erfahrung des Fremden in der deutschsprachigen Literaturnach 1945" bildeten drei literarische Werke den Ansatzpunkt für Diskussionen: Barbara Frischmuths Das Verschwinden des Schattens in der Sonne, Sten Nadolnys Selim oder die Gabe der Rede und Adolf Muschgs Im Sommer des Hasen. Bei diesem Seminar wurde der interkulturelle Aspekt ausnahmsweise nicht aus der deutsch-tschechischen Perspektive gedeutet.

- Das folgende Seminar fand in Regensburg statt: „Deutsch-tschechische Erzählweisen. Libuše Moníková". Die Teilnehmer setzten sich mit der Art und Weise auseinander, in der sich die Verbindungen zwischen der deutschen „Erzählsprache“ und dem tschechischen Kontext in den Werken von Moníková manifestieren.

- Für das nächste Treffen wurden Werke aus dem Bereich der deutschsprachigen Lyrik gewählt und das Thema einigermaßen allgemein formuliert: Lyrik in der Landeskunde. Erörtert wurden bei diesem Seminar die lyrischen Werke von J. W. von Goethe (Der Erlkönig), F. Schiller (Der Handschub), Ch. F. Hebbel (Der Heideknabe), C. F. Meyer (Die Füße im Feuer), T. Fontane (Die Brück' am Tay), F. Wedekind (Der Tantenmörder), P. Celan (Todesfuge). 
In den folgenden Jahren wurden in den Seminaren nicht nur literarische Akzente gesetzt, sondern z. B. die Sprache der Reklame in Form einer interkulturellen Gegenüberstellung untersucht.

\section{Mehrsprachigkeit und der literarische Kanon}

Versteht man unter Kanon „in der Literatur eine Auswahl mustergültiger Autoren und Werke [...]" (Wilpert 2001: 396), kann man bei der Gestaltung eines auf Mehrsprachigkeit abzielenden Literaturunterrichts verschiedene Vorgehensweisen bei der Kanonbildung befolgen. Demnach kann ein Kanon der literarischen Mehrsprachigkeit folgendermaßen strukturiert werden:

- Auswahl von Autoren, die neben Texten in ihrer Muttersprache auch Texte in einer Fremdsprache (oder mehreren Fremdsprachen) verfassen;

- Auswahl von Autoren, die Texte in ihrer Muttersprache verfassen, sie aber in einen deutlich interkulturellen Kontext setzen;

- Auswahl von Autoren, die bei der Niederschrift eines literarischen Werkes von mehreren Sprachen Gebrauch machen;

- Auswahl von einzelnen literarischen Werken, die ebenfalls den o. a. Merkmalen Rechnung tragen.

Bei der Suche nach Autoren und Werken, die einem „Kanon der literarischen Mehrsprachigkeit" genügen, kann man im Bereich der deutschsprachigen Literatur in jeder literarischen Epoche hinreichende Beispiele von literarischen Werken entdecken, die einem literarischen multilingualen Modell gerecht werden. Diese Tatsache kann auf folgende Ursachen zurückgeführt werden:

- Unstabile politische Lage (Nationalsozialismus, Krieg, Exil in seiner Europaund Überseephase, „Exil“ nach dem Jahre 1945),

- Migration (Situation nach dem Zweiten Weltkrieg - italienische, türkische Gastarbeiter),

- Divergenzen im religiösen Bereich (Luther und seine Übersetzertätigkeit),

- Existenz von deutschsprachigen Enklaven in Polen, Rumänien, Böhmen, Mähren, der Slowakei u. a. (Prager deutsche Literatur, deutschsprachige Autoren in Mähren, Rumänien, Bulgarien und anderen Ländern).

Die Merkmale einer literarischen Mehrsprachigkeit, ihre Form und Funktion, können transparent am Beispiel des literarischen Schaffens von Thomas, Heinrich und Klaus Mann gezeigt werden. Die älteste Generation der Buddenbrooks, die beim Sprechen französische Wörter verwendet, das Sprachenlabyrinth im Zauberberg, der französische geschichtliche Hintergrund im Schaffen von Heinrich Mann (HenriQuatre-Romane) oder die englisch geschriebenen Werke Klaus Manns aus seinem amerikanischen Exil sind nur einige Beispiele dafür, dass man im Bereich der deutschsprachigen Literatur unzählige Belege eines mehrsprachlichen Hintergrunds finden kann. 
Die mehrsprachigen Akzente können im Literaturunterricht auch an einem einzigen ausgewählten Werk gezeigt und diskutiert werden. Dem Unterrichtenden steht eine ganze Reihe von literarischen Texten zur Auswahl, die in einer einleitenden Unterrichtsstunde als ein minimalistischer Einstieg in die Problematik der Mehrsprachigkeit herangezogen werden können. Als Beispiel hierfür kann das Gedicht Come together von Gino Chiellino dienen, in dem die globale Wirkung der Reklamesprache in den Vordergrund gerückt wird:

come together

nel Mondo dei colori di Benetton

and learn to live as friends

im Lande der Nichtraucher wo die Fremde

wie Farben von Benetton geraucht wird

(Chiellino 1992: 77)

Abschließend soll an einigen Textproben aus der Erzählung Simultan von Ingeborg Bachmann gezeigt werden, dass sich die literarische Mehrsprachigkeit auf mehr semantischen Ebenen realisiert, als nur auf einer rein sprachlichen („multilingualen“).

In Simultan bildet die Mehrsprachigkeit nicht lediglich den Hintergrund der Handlung, sie ist vielmehr allgegenwärtig, durch eine simple Figurenkonstellation in den Mittelpunkt gerückt. Die Frauenfigur, aus deren Perspektive das Geschehen betrachtet wird, heißt Nadja. Dagegen wird der männliche Protagonist konsequent nur unpersönlich „Mr. Frankel“ genannt und Nadja wehrt sich sogar dagegen, seinen Vornamen Ludwig auszusprechen. Als sollte der o. a. Definition von Mehrsprachigkeit Genüge getan werden, können die Protagonisten von Deutsch zu Französisch, Englisch und Italienisch „ohne weiteres“ umschalten:

„Im Fahren hatten sie wenig miteinander reden können [...], nur vor der Ausfahrt in Salerno [...] gab es dies und jenes zu bemerken, einmal französisch, dann wieder englisch, italienisch konnte er noch nicht besonders gut, und mit der Zeit nahm sie den alten Singsang wieder an, sie melodierte ihre deutschen Sätze und stimmte sie auf seine nachlässigen deutschen Sätze ein, wie aufregend, daß sie wieder so reden konnte, nach zehn Jahren..."

(Bachmann 1972: 8)

Die sprachliche Vielfalt steht in dieser Erzählung stellvertretend nicht nur für den Verlust der gemeinsamen „Heimat“ Wien, sondern auch für den Verlust der Kontaktund Erinnerungsfähigkeit des Paares. Zu einer neuen Heimat wurde für beide ein globaler Raum, dessen Existenz in der Zeit der Genfer Abrüstungskonferenzen des Kalten Krieges noch nicht allerorts präsent war, wie es heutzutage der Fall ist. In der folgenden Textprobe wird der Sprachwechsel als ein Begleitphänomen der ansetzenden „Globalisierung" dargelegt: 
„Er war einige Jahre lang in Rourkela gewesen und zwei Jahre in Afrika, in Ghana, dann in Gabun, länger in Amerika selbstverständlich, [...] sie irrten beide die halbe Welt ab [...] und am Ende wußten sie ungefähr, [...] wo sie gedolmetscht und er etwas erforscht hatte $[\ldots]$ und sie kehrten aus Indien wieder nach Genf zurück [...]. Die Städte wirbelten auf in der Nacht, Bangkok, London, Rio, Cannes, dann wieder Genf unvermeidlich, Paris auch unvermeidlich. Nur San Francisco, das bedauerte sie lebhaft, no, never und gerade das hatte sie sich immer gewünscht, after all those dreadful places there, und immer nur Washington, grauenhaft, [...] dann schwiegen sie, ausgelaugt, und nach einer Weile stöhnte sie ein wenig, please, would you mind, je suis terriblement fatiguée, mais quandmême, c'est drôle, n'est-ce pas, d'être parti ensemble, tu trouves pas? I was flabbergasted when Mr. Keen asked me, no, of course not, I just call him Mr. Keen, denn er schien immerzu keen auf etwas zu sein, auch auf sie während der Party im Hilton, but let's talk about something more pleasant, I utterly disliked him.“ (Bachmann 1972: 9-10)

Die Unfähigkeit zu kommunizieren ist nicht auf ein erlernbares oder angeborenes Sprachsystem zurückzuführen. Während Mr. Frankel an verschiedenen Textstellen Nadja zweimal die Frage stellt, ob es „einmal eine einzige Sprache geben [werde]“ (Bachmann 1972: 15 und 29), ahnt Nadja, dass es zu einer einzigen Sprache, zu einem Daheim, keinen Weg mehr gibt:

„[...] und nun gar reisen, mit jemand aus Wien! Sie wußte bloß nicht, was sie deswegen einander zu sagen hatten, nur weil sie beide aus dieser Stadt kamen und eine ähnliche Art zu sprechen und beiseite zu sprechen hatten, vielleicht hatte sie auch nur [...] geglaubt, er bringe ihr etwas zurück, einen vermißten Geschmack, einen fehlenden Tonfall, ein geisterhaftes Gefühl von einem Daheim, das nirgends mehr für sie war." (Bachmann 1972: 8)

\section{Schlussbetrachtung}

Die wenigen hier vorgestellten Konzepte eines Literaturunterrichts, bei dem das mehrsprachliche Vorwissen der Studierenden im Lehrplan berücksichtigt wird, wollen ein konziser Beitrag zur Diskussion sein, welchen Unterschied es zwischen der realen Mehrsprachigkeit, die als vorhandene Kenntnis von einer oder mehreren Sprachen neben der Muttersprache verstanden wird, und der literarischen Mehrsprachigkeit gibt, die das Kommunizieren nicht nur als eine Verwirklichung des Sprechakts betrachtet, sondern die vielen Ebenen aufzudecken versucht, die sich hinter dem (Nicht-) Kommunizieren verbergen können. Die im Beitrag gezeigten Unterrichtskonzepte lassen die Vermutung zu, dass man im Falle der literarischen Mehrsprachigkeit viel differenzierter an den (literarischen) Text herangehen muss, als wenn man sich „nur“ mit dem Vergleich der Lexik von zwei oder mehreren verschiedenen Sprachen befasst. 


\section{Literaturverzeichnis}

Abraham, Ulf / Kepser, Matthis (2009): Literaturdidaktik Deutsch. Eine Einführung. Berlin, Schmidt.

Bachmann, Ingeborg (1972): Simultan. München, Piper.

Belke, Gerlind (2008): Mehrsprachigkeit und Deutschunterricht. Innsbruck, Studien.

Bredella, Lothar (2002): Literarisches und interkulturelles Verstehen. Tübingen, Narr.

Chiellino, Gino (1992): Sich die Fremde nehmen. Gedichte. Kiel, Neuer Malik Verlag.

Dannerer, Monika / Mauser, Peter / Scheutz, Hannes / Weiss, Andreas E. (Hg.) (2009): Gesprochen - geschrieben - gedichtet. Variation und Transformation von Sprache. Berlin, Schmidt.

Dohrn, Antje (2007): Leseförderung mit literarischen Texten im DaZ-Unterricht. Bausteine für einen integrativen Deutschunterricht. Frankfurt am Main, Lang.

Dawidowski, Christian / Wrobel, Dieter (Hg.) (2006): Interkultureller Literaturunterricht. Konzepte-Modelle-Perspektiven. Baltmannsweiler, Schneider Verlag Hohengehren.

Esselborn, Karl (2010): Interkulturelle Literaturvermittlung zwischen didaktischer Theorie und Praxis. München, Iudicium.

Griesmayer, Norbert (2000): Jenseits von Babylon. Innsbruck [u.a.], Studien.

Kriegleder, Wynfrid (2011): Eine kurze Geschichte der Literatur in Österreich. Menschen Bücher - Institutionen. Wien, Praesens.

Magris, Claudio (1966): Der habsburgische Mythos in der österreichischen Literatur. Salzburg, Otto Müller.

Martini, Fritz (1984): Deutsche Literaturgeschichte von den Anfängen bis zur Gegenwart. Stuttgart, Alfred Kröner.

Meurer, Petra / Ölke, Martina / Wilmes, Sabine (Hg.) (2009): Interkulturelles Lernen. Bielefeld, Aisthesis.

Multikulturalität, Interkulturalität, Transkulturalität und Plurikulturalität. http://www.ikud. de/Multikulturalitaet-Interkulturalitaet-Transkulturalitaet-und-Plurikulturalitaet.html (2. 4. 2012).

Neumann, Ursula (2009): Schulen in ,transnationalen Lebenswelten'. Ansätze im Modellprogramm FörMig-Foderung von Kindern und Jugendlichen mit Migrationshintergrund. In: Dartan, Muzaffer / Elis, Ali (Hg.): Migration - Bildung Integration? Dokumentation des gleichnamigen Symposiums über die Bildungschancen von Kindern und Jugendlichen mit Migrationshintergrund am 14. November 2008 in Bremen. Bremen, TDI Publikationen. S. 27-53.

Neumann, Ursula / Schneider, Jens (Hg.) (2011): Schule mit Migrationshintergrund. Münster [u.a.], Waxmann.

Oksaar, Els (1980): Mehrsprachigkeit, Sprachkontakt, Sprachkonflikt. In: Nelde, P. H. (Hg.): Sprachkontakt und Sprachkonflikt. Zeitschrift für Dialektologie und Linguistik. 11/Beiheft 32. S. 43-52.

Oksaar, Els (1988): Fachsprachliche Dimensionen. Tübingen, Narr.

Riehl, MariaClaudia (2008): Mehrsprachigkeit. Grundlagen, Vorteileunddidaktische Konsequenzen. http://www.uni-koeln.de/sdd2008/Riehl_Mehrsprachigkeit.pdf (25. 4. 2012).

Riehl, Claudia Maria (2009): Diskursmarkierung im mehrsprachigen Dialog. In: Dannerer, Monika / Mauser, Peter / Scheutz, Hannes / Weiss, Andreas E.Dannerer, Monika / Mauser, Peter / Scheutz, Hannes / Weiss, Andreas E. (Hg.)(2009): Gesprochen - geschrieben gedichtet. Variation und Transformation von Sprache. Berlin, Erich Schmidt. S. 205-222.

Rück, Nicola (2009): Auffassungen vom Fremdsprachenlernen monolingualer und plurilingualer Schülerinnen und Schüler. Kassel, Kassel UP. 
Wilpert, Gero von (2001): Sachwörterbuch der Literatur. Stuttgart, Kröner.

Wintersteiner, Werner (2006a): Poetik der Verschiedenheit. Literatur, Bildung, Globalisierung. Klagenfurt/Celovec, Drava.

Wintersteiner, Werner (2006b): Transkulturelle literarische Bildung. Die „Poetik der Verschiedenheit" in der literaturdidaktischen Praxis. Innsbruck [u.a.], Studien.

Žmegač, Viktor (Hg.) (1978): Geschichte der deutschen Literatur vom 18. Jahrhundert bis zur Gegenwart. Königstein, Athenäum. 